7-13-2020

\title{
Reliability of the Styku 3D Whole Body Scanner for the Assessment of Body Size in Athletes
}

Joe D. DeRouchey

Grant R. Tomkinson

University of North Dakota, grant.tomkinson@und.edu

Jesse L. Rhoades

jesse.rhoades@und.edu

John S. Fitzgerald

University of North Dakota, john.s.fitzgerald@UND.edu

\section{How does access to this work benefit you? Let us know!}

Follow this and additional works at: https://commons.und.edu/ehb-fac

Part of the Sports Sciences Commons

\section{Recommended Citation}

Joe D. DeRouchey, Grant R. Tomkinson, Jesse L. Rhoades, et al.. "Reliability of the Styku 3D Whole Body Scanner for the Assessment of Body Size in Athletes" (2020). Education, Health \& Behavior Studies Faculty Publications. 61.

https://commons.und.edu/ehb-fac/61

This Article is brought to you for free and open access by the Department of Education, Health \& Behavior Studies at UND Scholarly Commons. It has been accepted for inclusion in Education, Health \& Behavior Studies Faculty Publications by an authorized administrator of UND Scholarly Commons. For more information, please contact und.commons@library.und.edu. 


\title{
Reliability of the Styku 3D Whole Body Scanner for the Assessment of Body Size in Athletes
}

Running Head: RELIABILITY OF 3D ANTHROPOMETRY

Authors: Joe D. DeROUCHEY ${ }^{1}$, Grant R. TOMKINSON ${ }^{1,2}$, Jesse L. RHOADES ${ }^{1}$, and John S. FITZGERALD $^{1 *}$

\author{
Affiliations \\ ${ }^{1}$ Human Performance Laboratory, Department of Education, Health and Behavior Studies, \\ University of North Dakota, Grand Forks, ND, USA; \\ ${ }^{2}$ Alliance for Research in Exercise, Nutrition and Activity (ARENA), School of Health Sciences, \\ University of South Australia, Adelaide, SA, Australia
}

\section{Corresponding author}

Dr. John S. Fitzgerald*

$\bowtie \quad$ Department of Education, Health and Behaviour Studies

University of North Dakota

$27512^{\text {nd }}$ Avenue North, Stop 8235

Grand Forks, ND, 58202, USA

畺 $\quad+1701-777-2988$

john.s.fitzgerald@und.edu 
The authors have no conflicts of interest or external funding sources to disclose.

Word count: 2959. 


\begin{abstract}
Anthropometry is important for predicting sport performance. While 3-dimensional (3D) body scanners increase the feasibility of anthropometric assessment, reliability data on athletes are lacking. The aim of this study was to determine the test-retest reliability of a portable, singlecamera 3D body scanning system (Styku S100) to assess circumferences, and whole-body and segmental surface areas and volumes of athletes. Forty-nine (19 male) athletes were scanned six times (two sessions of three scans). The Styku scanner demonstrated nearly perfect reliability. Systematic errors were negligible (mean standardized bias [95\%CI]: scan 1 vs. 4, 0.04 [0.02, 0.06]), random errors were negligible (mean standardized typical error [95\%CI]): scan 1 vs. 4 , $0.14[0.10,0.17])$, and test-retest correlations were nearly perfect (mean intra-class correlation coefficient [95\%CI]: scan 1 vs. 4, 0.98 [0.97, 0.99]). Single-camera 3D body scanning systems may provide practitioners and researchers with a feasible tool to evaluate body size and shape.
\end{abstract}

Keywords: 3D anthropometry, whole body measurement, kinathropometry, repeatability, sport 


\section{Introduction}

Researchers and practitioners, interested in sport performance and health, have used anthropometry — the surface measurement of skinfolds, lengths, breadths, and circumferences of the human body — for decades (Kerr, Ackland, \& Schreiner, 1995). Direct manual anthropometric assessment (e.g., through the use of skinfold calipers, circumference tapes, bone calipers) has traditionally been used due to the low cost, availability, and maintenance of equipment. However, this approach is invasive, has a high participant burden, requires a high level of tester expertise, and is unable to directly measure whole and segmental body surface areas and volumes (Kuehnapfel, Ahnert, Loeffler, \& Scholz, 2017). Advancements in anthropometry, such as 3-dimensional (3D) body scanning, have sped up and simplified anthropometric assessment (Bragança, Arezes, Carvalho, \& Ashdown, 2016), considerably reducing the participant burden and tester training requirements. Additionally, 3D scanning is less invasive as there is no need for physical contact and participants can be scanned without being viewed by testers.

Anthropometric measures can be used to predict sports performance (Brocherie et al., 2014), track changes in body size and shape over an athlete's competitive season, and inform important decisions related to rehabilitation (Kordi et al., 2019). 3D scanners provide practitioners with the ability to measure large samples quickly and less-invasively compared to traditional methods, making in-competition measurements more feasible (Schranz, Tomkinson, Olds, \& Daniell, 2010; Schranz, Tomkinson, Olds, Petkov, \& Hahn, 2012). Interestingly, 2-dimensional (e.g., cross-sectional areas) and 3D (e.g., volumes and surface areas) measures are generally better predictors of sporting success than are 1-dimensional measures (e.g., lengths, girths, breadths) 
(Schranz et al., 2010, 2012). Furthermore, 3D scanning was required to capture many of the largest anthropometric differences (i.e., volumes and surface areas) between elite athletes and the general population in Schranz et al. (2010), comparisons which are commonly used to establish the importance of an anthropometric trait for sporting success (e.g., greater importance is indicated by larger differences in magnitude and/or reduced variability).

In recent years, the cost of 3D scanners has significantly decreased, with commercially available, single-camera systems (e.g., the Styku S100 scanner) demonstrating high reliability and comparing favorably with dual-energy x-ray absorptiometry (DXA) for circumference and segmental volume measurements in clinical settings (Bourgeois et al., 2017). The Styku S100 scanner, which uses a single camera to emit harmless infrared light, is a portable 3D scanner, making it an appealing field measure for sport. However, previous reliability studies evaluating single camera systems have not recruited athletes (Bourgeois et al., 2017; Ng, Hinton, Fan, Kanaya, \& Shepherd, 2016; Silver \& Wilson, 2020), who tend to represent the extreme in muscularity, and the anthropometric dimensions reported have varied. Athletes' greater muscle mass may increase measurement error associated with the automatic landmark identification software (e.g., thighs touching, or upper arm and torso contact, during standard scanning pose). Furthermore, while a recent study has reported improvements in reliability when averaging multiple scans of younger adults (Silver \& Wilson, 2020), it is not known whether improvements in reliability gained from averaging scans is practically meaningful in athletes. Adoption of 3D anthropometry may become more applicable to coaches, practitioners, and researchers if less expensive, portable scanners demonstrate high reliability in athletes. 
The aim of this study was to determine the test-retest reliability of the Styku S100 scanner to assess circumferences, and whole-body and segmental surface areas and volumes of collegiate and recreational athletes.

\section{Materials and Methods}

\section{Participants}

Forty-nine collegiate and recreational athletes (mean \pm SD: age $22.7 \pm 3.3$ yrs, height $174 \pm 8 \mathrm{~cm}$, mass $75 \pm 14 \mathrm{~kg}$, BMI $24.4 \pm 3.4 \mathrm{~kg} / \mathrm{m}^{2}$; 30 females, 19 males; 17 Division I collegiate athletes, 17 CrossFit athletes, 15 kinesiology students) were recruited from the University of North Dakota and retained in the analysis. Two athletes were excluded for missing data. Participants were excluded if they were injured, had casts or braces appended to their body, or were unable to stand unsupported on a raised rotating platform. The University of North Dakota Institutional Review Board approved all testing procedures. Informed written consent was obtained from all participants before the start of the study.

\section{Procedures}

During a single visit to the University of North Dakota's Human Performance Laboratory, participants were scanned six times (two sessions of three scans) with a 5-minute break between the two sessions so as to create two separate testing sessions. Upon arriving at the laboratory, participants changed into form fitting underwear (briefs for men and briefs plus sports bra for women) and had their height measured with a stadiometer (Seca, Chino, CA) to the nearest 0.1 $\mathrm{cm}$ and their mass measured with a digital scale (Detecto, Webb City, MO) to the nearest $0.1 \mathrm{~kg}$. Participants were ushered to the scanning area where they were scanned using a Styku 3D S100 
whole body scanner, which was configured using manufacturer specifications. This 3D scanner comprised a turntable, a Microsoft Kinect V2 camera (Microsoft Corporation, Redmond, WA) enclosed in a lightweight aluminum stand, and the MyBodee measurement extraction software (Styku, Los Angeles, CA). Participants were asked to step onto the turntable and assume a standard scanning pose, where they stood still, with their feet on the marked foot prints, arms abducted $\sim 45^{\circ}$, hands closed into a fist, head in a horizontal plane, whilst they breathed normally and the turntable rotated $360^{\circ}$ for a duration of $\sim 35$ seconds. During this time, the scanning stand comprising the Kinect camera system projected a structured light pattern onto the participant, with the reflections captured as Cartesian coordinates. The MyBodee software uses recognition technology to automatically locate surface landmarks, which were used to extract circumferences, and whole-body and segmental surface areas and volumes.

Three scans were made of each participant with minimal time in-between scans. The turntable was returned to the proper starting position and the standard scanning pose was reset before each scan. Participants then stepped off the turntable and rested in a standing/seated position for 5 minutes before they were scanned another three times. A scan was repeated if excessive movement during the scan was observed or indicated (image distortion) during a quick inspection after each bout of scans. Scans were also inspected for image and circumference placement irregularities during data extraction. All measurement data for a single scan were excluded from the analysis if irregularities were detected by one researcher (J.D.). Athletes were excluded from the analysis if they had missing data for scans 1 or 4, or had irregularities in two or more scans $(n=2)$. The mean of two scans, instead of three scans, was used in the second reliability analysis for athletes with irregularities detected on one scan $(n=6)$. 


\section{Statistical Analyses}

Between-session reliability was examined by comparing scans 1 and 4 and by comparing the means of scans 1-3 and 4-6. Data were inspected and two volume measurements for separate scans were identified as extreme errors ( $>$ five times the mean plus standard deviation of the between-session difference scores) and removed due to undue influence on reliability statistics. Descriptive statistics were presented as means and standard deviations. Systematic (bias) error, random (within-subject) error, and test-retest correlation were used to quantify measurement reliability. Systematic error was quantified as the absolute and standardized difference in means; random error as the percent and standardized typical error; and test-retest correlation as the intraclass correlation coefficient (ICC). All calculations were performed using a publically available reliability calculator (Hopkins, 2015). To interpret the magnitude of bias and typical error, standardized effect sizes (ES) of 0.2, 0.5 and 0.8 were used as thresholds for small, moderate and large respectively, with $\mathrm{ES}<0.2$ considered to be negligible (Cohen, 1988). Percent typical error data were compared to international error standards recommended by the International Society for the Advancement of Kinanthropometry (i.e., technical error of measurement $\leq 1.5 \%$ ) as the criterion-referenced threshold (Stewart, Marfell-Jones, \& International Society for Advancement of Kinanthropometry, 2011). This threshold is appropriate when examining the typical error when systematic bias is negligible. To interpret the magnitude of correlation, ES of $0.1,0.3,0.5$, 0.7 and 0.9 were used as thresholds for low, moderate, high, very high and nearly perfect respectively, with $\mathrm{ES}<0.1$ considered to be negligible (Cohen, 1988). Ninety-five percent confidence intervals $(95 \% \mathrm{CI})$ were calculated for all variables. A threshold for (error-free) real change for a given measurement was calculated by multiplying the percent typical error by 1.645 
(90\% confidence) as per Hopkins (2000) to determine the percent change needed to confidently detect real changes. Next, the resultant product was multiplied by the mean of session 1 .

\section{Results}

Between-session reliability using a single scan

The Styku S100 scanner demonstrated nearly perfect between-session reliability when comparing scans 1 and 4. Systematic errors were negligible (mean standardized bias [95\%CI]:

$0.04[0.02,0.06])$, random errors were negligible (mean typical error [95\%CI]): percent, $1.5[1.0$, $2.0]$; standardized, $0.14[0.10,0.17])$ with more than half of the measures $(68 \%)$ demonstrating acceptable random error compared to international standards (Table 1). Test-retest correlations were nearly perfect (mean ICC [95\%CI]: 0.98 [0.97, 0.99]). Differences in mean systematic errors, random errors, and test-retest correlations between body dimension types (circumferences, surface areas, and volumes) were negligible.

Between-session reliability using the mean of three scans

The Styku S100 scanner demonstrated nearly perfect between-session reliability when comparing the means of scans 1-3 and scans 4-6. Systematic errors were negligible (mean standardized bias $[95 \% \mathrm{CI}]: 0.02[0.01,0.03]$ ), random errors were negligible (mean typical error [95\%CI]): percent, $0.9[0.6,1.2]$; standardized, $0.08[0.06,0.10])$ with most measures $(82 \%)$ demonstrating acceptable random error compared to international standards (Table 2). Test-retest correlations were nearly perfect (mean ICC [95\%CI]: 0.99 [0.99, 1.00]). Differences in mean systematic errors, random errors, and test-retest correlations between body dimension types were 
negligible. The differences between using a single scan and the mean of three scans for typical errors were negligible.

$* * *$ Tables $1 \& 2$ near here***

\section{Discussion}

The aim of this study was to determine the test-retest reliability of a commercially available, portable 3D body scanning system (Styku S100) in athletes. We observed negligible betweensession systematic and random errors, and nearly perfect test-retest correlations, with most random errors considered "acceptable" relative to international error standards (i.e., technical error of measurement $\leq 1.5 \%$ ) when using a single scan and when taking the mean of three scans. These findings indicate that a single scan is all that is required to reliably collect most circumferences, surface areas, and volumes for research and professional applications. However, a comparison of between-session random errors show some measurements (e.g., arm circumferences, surface area and volume) have higher typical errors and appear to benefit from averaging multiple scans. Thus, taking the average of three scans may be indicated when evaluating these measurements. Between-session standardized random errors indicate that the Styku S100 scanner can detect negligible differences between individuals when using a single scan and when taking the mean of three scans.

The random errors reported in our study compare favorably to those reported for Kinect-based multi-camera systems to extract circumference measurements of cylinders (Clarkson, Wheat, Heller, \& Choppin, 2016) and thigh volume in humans (Bullas, Choppin, Heller, \& Wheat, 2016; 
Clarkson et al., 2016), and single-camera systems evaluating multiple anthropometric measures in the general population (Bourgeois et al., 2017; Ng et al., 2016). Notably, the reliability of the Styku S100 scanner in our athlete sample was similar to Bourgeois et al. (Bourgeois et al., 2017), who reported comparable random errors across four circumferences (coefficient of variation $[\mathrm{CV}]$ range: $0.3-0.8 \%)$ and six volumes (CV range: $0.3-2.4 \%)$ using the same technology on apparently healthy adults from a clinical center. In addition, $\mathrm{Ng}$ et al, (2016) evaluated the reliability of the Fit3D Proscanner and reported random errors across six circumferences $(\mathrm{CV}$ range: $0.8-2.2 \%$ ), four volumes (CV range: $0.7-4.5 \%$ ), and four surface areas (CV range: $0.8-$ $3.5 \%$ ) consistent with the random errors in this study, especially those calculated from single scans. Together these findings suggest that the Styku S100 scanner, which is designed for portability and cost savings, is capable of capturing reliable 3D measurements in general and athlete populations.

Our reliability data can be used to determine the threshold for real or meaningful change when monitoring individual athletes. To determine the threshold for a given measurement, coaches and practitioners can multiply the percent typical error for a measurement by $1.5-2.0$ (roughly $84-$ 95\% confidence) as per Hopkins (2000) to determine the percent change needed to confidently detect real changes for an individual athlete. We have provided the $90 \%$ confidence threshold for real change in absolute values associated with taking a single scan (Table 1) and using the mean of three scans (Table 2) for our group of athletes. Our thresholds for real change were similar to those reported in Silver and Wilson (2020) for 21 circumferences when using the Styku S100 scanner to evaluate between-day reliability in younger adults. Also consistent with Silver and Wilson (2020) was the reduction in the threshold for real change when the mean of multiple 
scans was taken to calculate between-session reliability: the thresholds for most measures were reduced by $30-50 \%$ when averaging multiple scans compared to taking a single scan. Coaches, practitioners, and researcher should consider the anthropometric variables of interest, the expected change due to intervention, and the time burden associated with scanning and data processing when determining if measurements should be derived from a single scan or averaged across multiple scans. It should also be appreciated that taking multiple scans during a session may help protect against missing data for an athlete due to scanning irregularities (e.g., movement artefacts), which may not be detected by the tester during the session.

3D scanners provide an efficient and non-invasive means of obtaining anthropometric measurements (Schranz et al., 2010), suitable for mass surveillance (e.g., team sports, research, etc.), which have meaningful relationships with sports performance. Thigh circumferences and cross-sectional areas (calculated using circumference data) have exhibited strong associations with sprinting speed (Hermassi et al., 2018) and countermovement jump performance in elite athletes (Brocherie et al., 2014). Whole-body and segmental body volumes appear to predict elite rowing performance (Schranz et al., 2010, 2012). In addition, limb circumferences, or preferably direct volumes, can be used to estimate lean mass volume (predictor of maximal-intensity exercise performance) (Kordi et al., 2019) and asymmetry (Rauter, Vodicar, \& Simenko, 2017). Moreover, 3D scanning could be used to obtain whole body and regional body composition estimates using prediction equations after calibration with DXA (Ng et al., 2016). Tracking changes in whole body and lower-extremity lean mass using 3D scanning would be an inexpensive and safer alternative to DXA and magnetic resonance imaging, and could be used to 
evaluate anthropometric responses to training and detraining, with potential applications to performance enhancement and rehabilitation (Kordi et al., 2019).

This study is not without limitations. Participants were competitive and recreational athletes with a mean BMI indicative of normal weight status. Therefore, the results of this study may not be generalizable to athletes competing in certain sports with a high prevalence of obesity (e.g., American football). Extremes in standing height were not represented in this study, which may limit generalizability to athletes competing in sports where standing height is advantageous (e.g., basketball). We did not examine the reliability of sessions on separate days. Thus, our reliability statistics may underestimate day-to-day typical errors. Future investigations should examine the reliability of single-camera systems across greater time periods (e.g., eight hours, 24 hours) to investigate within-day (circadian) and between-day variability, and recruit athletes with greater weight status and standing height. Participants' arms are not supported during the Styku S100 scanning procedure, which may augment error associated with movement during and between scans. However, the Styku S100 exhibited lower CVs for most circumference and volume measurements when compared to the Fit3D Proscanner, which provides handholds for the participant (Bourgeois et al., 2017). Future research should assess the effect of participant instructions designed to minimize postural deviations between scans. Our study did not assess the accuracy of scanned measurements, though previous studies evaluating single-camera systems found good agreement among 3D scanners and DXA derived whole-body and segmental volumes $\left(\mathrm{R}^{2}=0.69-0.99\right)$, tape measure circumferences $\left(\mathrm{R}^{2}=0.71-0.96\right)$, and the Du Bois model of whole body surface area $\left(\mathrm{R}^{2}=0.97\right)$ (Bourgeois et al., 2017; $\mathrm{Ng}$ et al., 2016). 
In conclusion, the commercially available, single-camera Styku S100 scanner is highly repeatable, capable of identifying negligible changes between individuals, and exceeds international standards for precision for most measurements. Coaches, practitioners, and scientists could utilize this portable technology to quickly measure large groups (e.g., sporting teams) without the invasiveness or technical proficiency required for manual measurements (Schranz et al., 2010). A multitude of measurements can be extracted or calculated from the scans, which can be used for talent identification (Schranz et al., 2010, 2012) and tracking changes associated with exercise training (Kordi et al., 2019), with the aim of optimizing exercise prescription for performance and health.

\section{Acknowledgements}

The authors thank the athletes who volunteered to participate in this study. There were no conflicts of interest or external funding sources for this study.

\section{References}

Bourgeois, B., Ng, B. K., Latimer, D., Stannard, C. R., Romeo, L., Li, X., ... Heymsfield, S. B. (2017). Clinically applicable optical imaging technology for body size and shape analysis: Comparison of systems differing in design. European Journal of Clinical Nutrition, 71(11), 1329-1335. https://doi.org/10.1038/ejcn.2017.142 
Bragança, S., Arezes, P., Carvalho, M., \& Ashdown, S. P. (2016). Current state of the art and enduring issues in anthropometric data collection. DYNA, 83(197), 22-30. https://doi.org/10.15446/dyna.v83n197.57586

Brocherie, F., Girard, O., Forchino, F., Haddad, H. A., Santos, G. A. D., \& Millet, G. P. (2014). Relationships between anthropometric measures and athletic performance, with special reference to repeated-sprint ability, in the Qatar national soccer team. Journal of Sports Sciences, 32(13), 1243-1254. https://doi.org/10.1080/02640414.2013.862840

Bullas, A. M., Choppin, S., Heller, B., \& Wheat, J. (2016). Validity and repeatability of a depth camera-based surface imaging system for thigh volume measurement. Journal of Sports Sciences, 34(20), 1998-2004. https://doi.org/10.1080/02640414.2016.1149604

Clarkson, S., Wheat, J., Heller, B., \& Choppin, S. (2016). Assessment of a Microsoft Kinectbased 3D scanning system for taking body segment girth measurements: A comparison to ISAK and ISO standards. Journal of Sports Sciences, 34(11), 1006-1014. https://doi.org/10.1080/02640414.2015.1085075

Cohen, J. (1988). Statistical Power Analysis for the Behavioral Sciences (2nd ed). Hillsdale, NJ: Lawrence Erlbaum Associates, Publishers.

Hermassi, S., Schwesig, R., Wollny, R., Fieseler, G., van den Tillaar, R., Fernandez-Fernandez, J., ... Chelly, M.-S. (2018). Shuttle versus straight repeated-sprint ability tests and their relationship to anthropometrics and explosive muscular performance in elite handball players. The Journal of Sports Medicine and Physical Fitness, 58(11), 1625-34. https://doi.org/10.23736/S0022-4707.17.07551-X

Hopkins, W. G. (2000). Measures of Reliability in Sports Medicine and Science. Sports Medicine, 30(1), 1-15. https://doi.org/10.2165/00007256-200030010-00001 
Hopkins, W. G. (2015). Spreadsheets for analysis of validity and reliability. Retrieved December 9, 2017, from Sportscience website: https://www.sportsci.org/2015/ValidRely.htm

Kerr, D. A., Ackland, T. R., \& Schreiner, A. B. (1995). The elite athlete-Assessing body shape, size, proportion and composition. Asia Pacific Journal of Clinical Nutrition, 4(1), 25-29.

Kordi, M., Haralabidis, N., Huby, M., Barratt, P. R., Howatson, G., \& Wheat, J. S. (2019). Reliability and validity of depth camera 3D scanning to determine thigh volume. Journal of Sports Sciences, 37(1), 36-41. https://doi.org/10.1080/02640414.2018.1480857

Kuehnapfel, A., Ahnert, P., Loeffler, M., \& Scholz, M. (2017). Body surface assessment with 3D laser-based anthropometry: Reliability, validation, and improvement of empirical surface formulae. European Journal of Applied Physiology, 117(2), 371-380. https://doi.org/10.1007/s00421-016-3525-5

Ng, B. K., Hinton, B. J., Fan, B., Kanaya, A. M., \& Shepherd, J. A. (2016). Clinical anthropometrics and body composition from 3D whole-body surface scans. European Journal of Clinical Nutrition, 70(11), 1265-1270. https://doi.org/10.1038/ejcn.2016.109

Rauter, S., Vodicar, J., \& Simenko, J. (2017). Body Asymmetries in Young Male Road Cyclists. International Journal of Morphology, 35(3), 907-912. https://doi.org/10.4067/S071795022017000300018

Schranz, N., Tomkinson, G., Olds, T., \& Daniell, N. (2010). Three-dimensional anthropometric analysis: Differences between elite Australian rowers and the general population. Journal of Sports Sciences, 28(5), 459-469. https://doi.org/10.1080/02640411003663284

Schranz, N., Tomkinson, G., Olds, T., Petkov, J., \& Hahn, A. G. (2012). Is three-dimensional anthropometric analysis as good as traditional anthropometric analysis in predicting 
junior rowing performance? Journal of Sports Sciences, 30(12), 1241-1248. https://doi.org/10.1080/02640414.2012.696204

Silver, B., \& Wilson, P. B. (2020). Reliability and Minimal Detectable Change of the Styku 3D Body Scanner. Measurement in Physical Education and Exercise Science, 1-7. https://doi.org/10.1080/1091367X.2020.1751634

Stewart, A., Marfell-Jones, M., \& International Society for Advancement of Kinanthropometry. (2011). International standards for anthropometric assessment. Lower Hutt, New Zealand: International Society for the Advancement of Kinanthropometry. 